\title{
Estudo comparativo dos perfis antropométrico e fisiológico e avaliação isocinética da força muscular nos membros inferiores em triatletas portugueses Comparative study of anthropometric and physiological profiles and isokinetic muscle strength assessment in the lower limbs in Portuguese triathletes
}

Faber Sérgio Bastos Martins, D.Sc.*, José Manuel da Costa Soares, D.Sc.**

*Escola Superior de Educação de Fafe (ESEF), **Faculdade de Desporto - Universidade do Porto

\section{Resumo}

Objectivo: Analisar o perfil antropométrico e fisiológico dos triatletas portugueses e avaliar os parâmetros isocinéticos da força muscular nos membros inferiores. Material e métodos: A amostra foi composta de 12 atletas seniores masculinos (30,3 \pm 3,8 anos; $70,2 \pm 4,4 \mathrm{~kg} ; 177,5 \pm 5,4 \mathrm{~cm}$; IMC 22,3 $\pm 0,6 \mathrm{e}$ percentagem de gordura corporal $6,7 \pm 1,9 \%)$. Foram avaliadas a impulsão vertical (SCM e SE), potência anaeróbia lática (WingateTest), força muscular em dinamómetro isocinético (velocidades angulares $90 \%$ e $360 \%$ s), potência aeróbia máxima (tapete rolante). Utilizou-se a estatística descritiva, teste T de Student para medidas repetidas e coeficiente de correlação de Spearman. Resultados: Foram encontradas correlaçóes entre a massa corporal dos triatletas e a potência média $(\mathrm{r}=0,57, \mathrm{p}=0,05)$ e máxima $(\mathrm{r}=0,59, \mathrm{p}=0,04)$ obtidas no teste de Wingate. A potência média (relativa à massa corporal) correlacionou-se com os valores dos torques máximos concêntricos da articulação do joelho à $90 \% \mathrm{~s}(\mathrm{r}=0,683, \mathrm{p}=0,014)$ e $360 \% \mathrm{~s}(\mathrm{r}=0,622, \mathrm{p}=$ $0,031)$. Os triatletas revelaram diferenças de $5,5 \%(\mathrm{p}$ $=0,000)$ na razão I/Q à 360\% e de 7,3\% (p=0,001) e $9,9 \%(\mathrm{p}=0,002)$ nos torques máximos concêntricos à $90 \%$ s e $360 \%$ s, respectivamente. Conclusão: Triatletas portugueses possuem perfis similares aos dos triatletas internacionais e evidenciam diferenças nos parâmetros isocinéticos da força muscular nos membros inferiores.

Palavras-chave: atletas, triathlon, potência anaeróbia, potência aeróbia, dinamómetro.

Recebido em 10 de março de 2013; aceito em 16 de abril de 2013.

Endereço para correspondência: Faber Sérgio Bastos Martins, Instituto de Estudos Superiores de Fafe, Rua Universitária, Apartado 178,4824-909 Medelo Portugal, E-mail: fabermartins@iesfafe.pt, jmsoares@ fade.up.pt 


\section{Abstract}

Objective: To analyze the anthropometric and physiological profile of Portuguese triathletes and to evaluate the parameters of isokinetic muscle strength in the lower limbs. Methods: The sample was composed of 12 male senior athletes $(30.3 \pm 3.8$ years, $70.2 \pm 4.4$ $\mathrm{kg}, 177.5 \pm 5.4 \mathrm{~cm}$, BMI $22.3 \pm 0.6$ and fat mass $6.7 \pm$ $1.9 \%)$. We evaluated the vertical jump (SCM and SE), lactic anaerobic power (Wingate Test), muscle strength on an isokinetic dynamometer (angular velocity 90 $\%$ s and $360 \%$ s) and maximal aerobic power (treadmill protocol). We used descriptive statistics, Student $\mathrm{t}$ test for repeated measures and Spearman correlation coefficient. Results: We found correlation between the body mass of triathletes and average power $(r=0.57$, $\mathrm{p}=0.05)$ and maximum $(\mathrm{r}=0.59, \mathrm{p}=0.04)$ obtained in the Wingate test. The average power (relative to body mass) correlated with the values of peak torques concentric knee joint at $90 \% \mathrm{~s}(\mathrm{r}=0,683, \mathrm{p}=0.014)$ and $360 \%(\mathrm{r}=0,622, \mathrm{p}=0.031)$. The triathletes revealed differences of $5.5 \%(\mathrm{p}=0.000)$ in the ratio I/Q to $360 \%$ and $7.3 \%(\mathrm{p}=0.001)$ and $9.9 \%(\mathrm{p}=$ 0.002 ) in concentric peak torque at $90 \%$ and $360 \%$, respectively. Conclusion: Portuguese triathletes have similar profiles to those of international triathletes and show differences in parameters and isokinetic muscle strength in the lower limbs.

Key-words: athletes, triathlon, anaerobic power, aerobic power, dynamometer.

\section{Introdução}

A realização de esforços prolongados pressupóe a contribuiçáo de uma diversidade de fatores, os quais exercem influência relevante na capacidade de prestação do atleta, podendo as exigências metabólicas variar em função da intensidade e duração do esforço.

O triathlon contempla a combinaçáo de três disciplinas (natação, ciclismo e corrida), realizadas de forma contínua em regime aeróbio e conjugadas com duas transiçóes, exigindo dos seus praticantes o desenvolvimento da capacidade aeróbia [1,2]. Adicionalmente, em virtude da natureza do esforço predominante nas provas de triathlon, o qual se traduz numa exigência acentuada do metabolismo oxidativo, observam-se elevados valores de consumo máximo de oxigénio $\left(\mathrm{VO}_{2 \max }\right)$ e limiar anaeróbio $\left(\mathrm{ml} \cdot \mathrm{kg}^{-1} \cdot \mathrm{min}^{-1}\right.$ e $\%$ $\left.\mathrm{VO}_{2 \max }\right)$ [3-5].

Neste sentido, importa salientar que simultaneamente ao processo de treino corretamente delineado, o qual permite o desenvolvimento das adaptaçóes conotadas com o aumento do rendimento, o perfil antropométrico se apresenta como fator intrínseco determinante, possibilitando uma elevada eficiência mecânica nas modalidades cíclicas referidas [6]. A substantivar, refere-se a correlação significativa entre a percentagem de gordura corporal e a performance obtida pelos atletas de triathlon durante os Jogos Olímpicos [7], enquanto nas provas de ciclismo profissional, a massa corporal do ciclista pode responder por uma variabilidade de 15 a $20 \%$ na performance obtida [8]. A contribuição da aptidão aeróbia no desempenho de uma tarefa de carácter anaeróbio (potência anaeróbia máxima e capacidade anaeróbia) está bem estabelecida, sendo a sua correspondência de génese variável, podendo a participação dos diferentes sistemas energéticos estar associada à metodologia de treino e ao período de treino no qual o atleta se encontra [9].

Acresça-se quea deterioração da potência e força musculares, expressas na redução dos valores de força explosiva, está de acordo com a especificidade das adaptações do músculo esquelético induzida pelas cargas regulares e sistemáticas do treino de duração prolongada [10].Não obstante, o padrão motor de uma determinada atividade físico-desportiva poderá exercer uma influência significativa no perfil funcional do atleta. Neste sentido, a força muscular constitui um componente fundamental no desempenho desportivo, onde a correta funcionalidade dinâmica dos músculos estabilizadores das articulaçóes se mostra determinante na prevenção das lesôes dos diversos tecidos [11].

Todavia, importa aduzir o facto da suscetibilidade às lesôes se mostrar aumentada quando diagnosticados desequilíbrios musculares na razão agonista/antagonista, em particular quando mensurados níveis de força muscular a baixas velocidades angulares em dinamômetro isocinético[11,12]. Em conformidade com os pressupostos 
anteriormente referidos, suscita o interesse por conhecer o perfil fisiológico dos atletas praticantes de triathlon, o que possibilita uma melhor compreensão das respectivas variáveis intervenientes na otimização da performance.

\section{Material e métodos}

\section{Amostragem}

A amostra foi constituída por 12 atletas masculinos integrantes da Federaçáo Portuguesa de Triathlon, com idade média de 30,3 $\pm 3,8$ anos, massa corporal de 70,2 $\pm 4,4 \mathrm{~kg}$, estatura corporal de $177,5 \pm 5,4 \mathrm{~cm}$, índice de massa corporal de $22,3 \pm 0,8$ e percentagem de gordura corporal $6,7 \pm 1,9 \%$.

\section{Procedimentos}

- Antropometria - Para a determinação da estatura, massa e percentagem de gordura corporal dos atletas foram utilizados o estadiômetro (modelo Rudolf Martin) e a balança digital de bioimpedância (modelo Tanita TBF 305), respectivamente.

- Avaliação da impulsão vertical - Foram utilizadas as técnicas de salto vertical máximo com contramovimento (SCM) e a partir de uma posição estática (SE). Os testes foram realizados sobre uma plataforma resistiva conectada a um timer digital ( \pm 0,001s/ ergojump) (Digitest OY, Muurame, Finland), sendo o tempo de voo (TV) durante o salto e a altura atingida pelo centro de gravidade determinados através da equação: $h=g x T V^{2} / 8[13]$.

- Avaliação da potência anaeróbia máxima - Foi adotado o Wingate Anaerobic Cycle Test (WAnT), com duraçáo total de 30 segundos e uma carga correspondente à $75 \mathrm{~g} / \mathrm{kg}$ de massa corporal do atleta, sendo registados os indicadores: Potência Máxima (Wmax), Potência Média (Wmed), Potência Mínima (Wmin), expressos em termos absolutos (Watts) e relativos a massa corporal (Watts/ $\mathrm{kg}$ ) do triatleta avaliado e oÍndice de Fadiga (IF\%).

- Avaliação isocinética da força muscular nos membros inferiores - Foi determinada através do dinamómetro isocinético Biodex ${ }^{\mathrm{TM}}$ System 2 , sendo mensurados os valores dos torques máximo e mínimo concêntricos dos músculos extensores e flexores da articulação dojoelho, trabalho total realizado, potência média obtida nas velocidades angulares de $90 \%$ s $(1,57$ $\mathrm{rad} / \mathrm{s})$ e $360^{\circ} / \mathrm{s}$ (6,28 rad $\left./ \mathrm{s}\right)$. Os triatletas foram posicionados e estabilizados no dinamómetro, de forma a prevenir eventuais movimentos acessórios, sendo o eixo de rotaçáo do aparelho alinhado com a parte lateral do côndilo femoral e o braço de alavanca ajustado e fixado acima dos maléolos. Inicialmente foram avaliados os índices de força muscular durante a extensão e flexão do joelho à velocidade de angular de $360^{\circ} \%$, sendo realizadas 5 repetiçóes máximas com cada membro. A seguir ao intervalo de recuperação de 5 minutos, foi realizada a segunda avaliação, onde os triatletas efetuaram uma série de 20 repetiçóes máximas de extensão/flexão do joelho, agora a velocidade angular de $90 \%$ s.

- Avaliação da potência aeróbia máxima $\left(V O_{2 m a x}\right)$ - Foi utilizado o protocolo contínuo com velocidades crescentes, com velocidade inicial de $2,6 \mathrm{~m} / \mathrm{s}$ e o respectivo patamar de 2 minutos [14]. O coeficiente de inclinaçáo do tapete foi mantido em zero (0\%) durante toda a avaliação. Os demais patamares tinham a duração de 1 minuto e o acréscimo de velocidade de $1 \mathrm{~m} / \mathrm{s}$ por patamar. $\mathrm{O}$ consumo de oxigénio foi avaliado através do analisador de trocas respiratórias Cortex: Metalyser 3B. O limiar anaeróbio (Lan) foi determinado pelo método ventilatório $\mathrm{V}$ -Slope $\left(\mathrm{VCO}_{2} \mathrm{vs}_{2}\right)$ através do Software Meta Soft 2.6. Foram utilizadas comomedidas descritivas a média e o desvio-padráo. A comparaçáo das médias dos indicadores funcionais e fisiológicos foi efectuada através do Teste T de Student para medidas repetidas. Para verificar os níveis de associaçáo entre as variáveis investigadas no estudo, recorreu-se ao coeficiente de correlação de Spearman. Os níveis de significância foram mantidos em $5 \%(\mathrm{p}<0,05)$. Os procedimentos estatísticos foram tratados e analisados nos programas Excel $^{\mathrm{TM}} 2000$ e SPSS ${ }^{\mathrm{TM}} 16.0$. 


\section{Resultados}

Tabela I - Avaliação do teste de impulsão vertical no salto com contramovimento (SCM) e salto estático (SE).

\begin{tabular}{ccc}
\hline Triatletas & SCM & SE \\
\hline$X \pm D p$ & $31,0 \pm 2,8$ & $30,7 \pm 1,9$ \\
\hline
\end{tabular}

Verificou-se um valor médio ligeiramente superior na avaliação do salto com contramovimento $(31,0 \pm 2,8$ vs $30,7 \pm 1,9 \mathrm{~cm})$, embora a diferença entre os respectivos valores obtidos no teste de impulsão vertical não se mostrou estatisticamente significativa $(t=0,295$, $\mathrm{p}=0,773)$.

Observou-se que os valores referentes à potência máxima absoluta e relativa foram $730 \pm 68,7 \mathrm{~W}$ e 10,2 $\pm 0,9$ Watts $/ \mathrm{kg}$, respectivamente. Inerentes aos registros da potência média absoluta e relativa, verificaram-se os valores $578,5 \pm 80,8$ Watts e 8,1 $\pm 1,3$ Watts $/ \mathrm{kg}$, respectivamente. O índice de fadiga (IF), mensurado em termos percentuais, registou como valor médio $36,6 \pm 8,1 \%$.

Verificou-se,à velocidade angular de $90 \%$, uma diferença significativa $7,3 \%(t=4,842$ $\mathrm{p}=0,001)$ do torque máximo concêntrico da articulação do joelho direito em relação ao joelho esquerdo $(181,1 \pm 9,9$ vs $169,0 \pm 14,2$ $\mathrm{Nm}$, respectivamente). Igualmente notória e consequentemente significativa, a diferença de $9,9 \%(t=3,925, \mathrm{p}=0,002)$ constatada entre os valores registados pelos músculos extensores $\mathrm{da}$ articulação do joelho direito em relaçáo ao joe- lho esquerdo $(106,6 \pm 12,3$ vs $97,0 \pm 12,8 \mathrm{Nm}$, respectivamente) a velocidade angular de $360 \%$ s.

Embora registados valores superiores para a flexão da articulação do joelho direito em relação ao joelho esquerdo, as velocidades angulares de $90 \%$ e $360 \%$ simporta aduzir a ausência da relevância estatística nas respectivas diferenças bilaterais de $6,9 \%(\mathrm{t}=2,133, \mathrm{p}=0,056)$ e $7 \%$ $(\mathrm{t}=0,833 \mathrm{p}=0,422)$.

Figura 1 - Valores (\%) da razão da força muscular Isquiotibiais/Quadriceps (I/Q) dos triatletas avaliados às velocidades angulares de $90 \% \mathrm{se} 360 \%$ s.

Razão I/Q -Velocidade Angular - 90\% (\%)
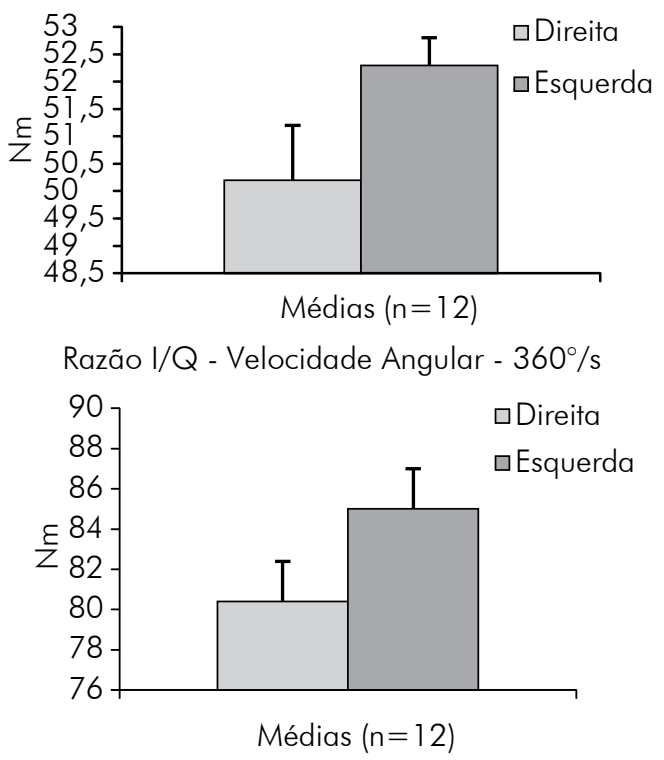

Tabela II - Valores de potência máxima (Wmax), potência média (Wmed), potência minima (Wmin) e indice de fadiga (I F \%) obtidos no Teste Wingate.

\begin{tabular}{lccccccc}
\hline Triatletas & $\mathrm{W}($ max $)$ & $\mathrm{Wmax} / \mathrm{kg}$ & $\mathrm{W}(\mathrm{med})$ & $\mathrm{Wmed} / \mathrm{kg}$ & $\mathrm{W}(\mathrm{min})$ & $\mathrm{Wmin} / \mathrm{kg}$ & $\mathrm{IF}(\%)$ \\
\hline $\mathrm{X} \pm \mathrm{Dp}$ & $730 \pm 68,7$ & $10,2 \pm 0,9$ & $578,5 \pm 80,8$ & $8,1 \pm 1,3$ & $461,3 \pm 97,1$ & $6,5 \pm 1,7$ & $36,6 \pm 8,1$ \\
\hline
\end{tabular}

Tabela III - Valores do torque máximo concêntrico $(\mathrm{Nm})$ do músculo quadriceps dos triatletas avaliados às velocidades angulares de $90 \%$ e $360 \%$ s.

\begin{tabular}{ccccc}
\hline Triatletas & Dir. $\left(90^{\circ} / \mathrm{s}\right)$ & Esq. $\left(90^{\circ} / \mathrm{s}\right)$ & Dir. $\left(360^{\circ} / \mathrm{s}\right)$ & Esq. $\left(360^{\circ} / \mathrm{s}\right)$ \\
\hline $\mathrm{X} \pm \mathrm{Dp}$ & $181,1 \pm 9,9$ & $169 \pm 14,2$ & $106,6 \pm 12,3$ & $97 \pm 12,8$ \\
\hline
\end{tabular}

Tabela IV - Valores do torque máximo concêntrico $(\mathrm{Nm})$ dos músculos isquiotibias dos triatletas avaliados às velocidades angulares de $90 \% \mathrm{~s}$ e $360 \%$.

\begin{tabular}{ccccc}
\hline Triatletas & Dir. $\left(90^{\circ} / \mathrm{s}\right)$ & Esq. $\left(90^{\circ} / \mathrm{s}\right)$ & Dir. $\left(360^{\circ} / \mathrm{s}\right)$ & Esq. $\left(360^{\circ} / \mathrm{s}\right)$ \\
\hline $\mathrm{X} \pm \mathrm{Dp}$ & $93,6 \pm 14,4$ & $89,2 \pm 14,2$ & $84,3 \pm 12,6$ & $82,4 \pm 13,6$ \\
\hline
\end{tabular}


A razão I/Q apresentou, à velocidade angular de $90 \%$, uma diferença bilateral não significativa de 4,1\% $(\mathrm{t}=-1,260, \mathrm{p}=0,234)$. Todavia, quando mensurada a velocidade de $360 \%$, registrou-se uma diferença estatisticamente significativa de $5,5 \%(\mathrm{t}=-4,947, \mathrm{p}=0,000)$ no grupo de triatletas avaliados.

Tabela V - Valores de consumo máximo de oxigénio absoluto (l/min) e relativo ( $\mathrm{ml} / \mathrm{kg} / \mathrm{min}$ ) obtidos noprotocolo de mensuração doVO ${ }_{2 \max }$ realizado em tapete rolante.

\begin{tabular}{ccc}
\hline Triatletas & \multicolumn{2}{c}{$\begin{array}{c}\text { Consumo Máximo de Oxigénio } \\
\left(\mathrm{VO}_{2 \text { max }}\right)\end{array}$} \\
\hline & $\begin{array}{c}\text { Absoluto } \\
(\mathrm{l} / \mathrm{min})\end{array}$ & $\begin{array}{c}\text { Relativo } \\
(\mathrm{ml} / \mathrm{kg} / \mathrm{min})\end{array}$ \\
\hline $\mathrm{X} \pm \mathrm{Dp}$ & $4,5 \pm 0,6$ & $64,9 \pm 7,7$ \\
\hline
\end{tabular}

\section{Discussão}

Os indicadores antropométricos avaliados mostram que os dados obtidos corroboram resultados de estudos anteriores realizados com propósitos similares e análogas amostras $[6,7,15]$. Em linha de convergência, importa referir a similaridade dos valores referentes a massa corporal dos triatletas com os valores médios registados por ciclistas contra-relogistas de elevada performance [16].

A substantivar, salienta-se a significativa correlaçâo entrea massa corporaldos triatletas e os valores de potência máxima $(\mathrm{r}=0,57, \mathrm{p}=0,05)$ e potência média $(\mathrm{r}=0,59, \mathrm{p}=0,4)$, obtidos na avaliação da capacidade anaeróbia em cicloergômetro, corroborando a influência deste indicador antropométrico na performance do WingateTest [17].

O valor médio registrado na estatura corporal encontra-se ligeiramente inferior ao reportado por estudos similares com triatletas de elite $[3,6,7]$. Evidências apresentadas em estudos com triatletas parecem demonstrar a vantagem de uma maior estatura corporal quando da realizaçáo de esforços de modalidades cíclicas como a natação e a corrida [18].

Neste sentido, e nas provas de triathlon em particular, ao adotarmos como constante a aplicação das forças, os segmentos corporais maiores permitem uma maior amplitude dos movimen- tos, reduzindo no segmentoa frequência gestual para uma determinada velocidade, fato que resulta numa maior economia de esforço para os respectivos valores de consumo de oxigénio e concentração de lactato sanguíneo [19].

Em conformidade com o postulado pelos referidos autores, uma elevada frequência gestual implica num aumento mais pronunciado das concentraçôes de lactato, evidenciando, desta forma, a importância de retardar os efeitos da fadiga através de uma maior eficiência motora atribuída a vantagem antropométrica do atleta.

Adicionalmente, a contribuição de um maior segmento corporal representa um importante fator intrínseco na economia de movimento, o qual se traduz numa vantagem hidrodinâmica em nadadores, onde uma elevada razão envergadura/ altura e elevada razão diâmetro bi-acromial/diâmetro bi-cristal constitui um menor coeficiente de arrasto, optimizando a performance na natação [6].

De acordo com Swain [8], um atleta de estatura superior possui uma vantagem nas provas de potência, podendo beneficiar-se da utilização de desmultiplicações mais elevadas a altas rotaçóes no ciclismo, embora dada a sua maior superfície corporal, acresça a sua respectiva resistência ao deslocamento. Os reduzidos valores de percentagem da gordura corporal registrados no presente estudo corroboram os valores encontrados em triatletas internacionais de elevada prestação $[6,7,15,18]$, valorizando funcionalmente o poder diferenciador deste indicador antropométrico em atletas de esforços prolongados.

Uma elevada percentagem de gordura corporal resulta no aumento do isolamento térmico corporal do atleta, implicando num aumento da temperatura interna, o que resulta numa resposta hemodinâmicade forma a desviar um maior fluxo para a superfície cutânea, reduzindo a perfusão sanguínea para o tecido ativo e consequente redução da performance aeróbia [18].

Face aos dados registrados na avaliação da impulsão vertical dos triatletas e tendo por referência os valores obtidos com atletas das respectivas modalidades singulares, observa-se que os valores médios dos saltos SCM e SE são inferiores aos encontrados em corredores de meio-fundo curto $(38,3 \pm 5,2 \mathrm{~cm}$ e $36,0 \pm 3,6 \mathrm{~cm}$, respectivamen- 
te) sendo, no entanto, mais elevados quando comparados com atletas de elite das provas de meio-fundo longo $(27,6 \pm 2,7 \mathrm{~cm}$ e $25,7 \pm 2,7$ $\mathrm{cm}$, respectivamente).

Estes resultados nos permitem sugerir que os triatletas podem possuir valores superiores aos atletas do meio-fundo longo em resposta a exigência imposta pelo treino de ciclismo que, sendo um exercício concêntrico na sua totalidade, requer constantes alteraçóes nos padróes de recrutamento das fibras musculares, o que provavelmente resulta numa maior solicitação das fibras musculares de contracção rápida. Nesta perspectiva, Komi et al. [20] referem que corredores das provas de meio-fundo curto apresentam uma maior percentagem de fibras de contracção rápida $(47,8 \%)$ comparativamente aos corredores do meio-fundo longo (34.6\%). Adicionalmente, a avaliaçáo da força explosiva pode também ser estudada através do chamado índice de elasticidade $(\Delta \mathrm{h})$ que resulta da diferença encontrada a partir dos valores obtidos no SCM e SE, o qual nos permite conhecer o potencial elástico dos músculos.

Os dados registados pelos triatletas nos respectivos saltos evidenciam um valor de $\Delta \mathrm{h}$ baixo (0.3), podendo este dever-se a uma possível deterioração dos níveis de força muscular em consequência da especialização competitiva, centrada nas sucessivas cargas de treinode corrida contínua em regime aeróbio que, pela sua duração e periodicidade, reflecte-se na diminuição significativa das qualidades contráteis explosivas das fibras musculares [21].

Em sintonia com o resultado de estudos anteriores [22], em que foi avaliada a contribuição dos diferentes segmentos corporais na elevação do centro de gravidade do corpo humano, foi evidenciada no presente estudo uma correlação moderada $(r=0,587, p=0,045)$ entre os valores registados do salto SCM e o torque máximo concêntrico da articulação do joelho à velocidade de $90 \%$ s. A referida correlação parece justificar-se no fato do movimento de extensão da articulação do joelho contribuir com $56 \%$ da velocidade do salto, influenciando de forma significativa o seu desempenho [22].

A análise atinente à avaliação da capacidade anaeróbia lática, mensurada em cicloergômetro (Wingatetest), permite a constatação de uma simi- litude nos registos das potências máxima e média pelos presentes triatletas quando comparados com triatletas competitivos internacionais [23-25].

Foram encontradas correlaçôes significativas entre o valor registrado da potência média relativa à massa corporal dos triatletas e os valores referentes aos torques máximos concêntricos $\mathrm{da}$ articulação do joelho às velocidades angulares de $90 \% \mathrm{~s}(\mathrm{r}=0,683, \mathrm{p}=0,014)$ e $360 \% \mathrm{~s}(\mathrm{r}=0,622$, $\mathrm{p}=0,031)$, corroborando assim os resultados obtidos em estudos similares com atletas de outras modalidades desportivas [26,27].

A literatura mostra-se concordante no que respeita a possibilidade acrescida de uma irregularidade muscular e consequente aumento do risco de lesōes articulares, musculares e tendinosas, quando mensuradas diferenças bilaterais de força muscular superiores a $15 \%[28,29]$.

Pese embora uma leitura de valores que possam realçar o caráter bilateral na presente amostra, não se vislumbrou a constatação de diferenças estatisticamente significativas, estando os resultados obtidos inseridos nos valores de referência sugeridos pelos autores. Neste sentido, importa considerar que as diferenças bilaterais $\mathrm{da}$ força muscular assim como a relaçâo I/Q estão relacionadas com as exigências particulares dos respectivos desportos, podendo o padráo motor de uma determinada modalidade exercer uma influência relevante no perfil funcional do atleta [29]. Relativamente à razão $\mathrm{I} / \mathrm{Q}$, mensurada as velocidades angulares de $90 \%$ e $360 \%$ s,salienta-se que os valores registados encontram-se em consonância com os valores de referênciacontemplados na literatura [29].

No âmbitoda avaliação da potência máxima aeróbia $\left(\mathrm{VO}_{2 \max }\right)$ realizada em ergómetro (tapete rolante), o valor médio obtido pelos triatletas $(64,9 \pm 7,7 \mathrm{ml} / \mathrm{kg} / \mathrm{min})$ corrobora os resultados de triatletas internacionais masculinos competitivos[30-32], embora se mostre inferior aos valores médios encontrados em triatletas de elite de provas olímpicas $[7,33,34]$.

$\mathrm{O} \mathrm{VO}_{2 \max }$ constitui um importante preditor da performance em provas de corrida, apresentando uma elevada correlaçáo com o tempo final em provas de triathlon $[2,4,7,18]$. Todavia, no que concerne à performance em esforços prolongados, os indicadores respirató- 
rios, circulatórios e metabólicos, determinados a intensidades submáximas, mostram-se mais sensíveis, reforçando assim o limiar anaeróbio como um critério mais consistente na avaliaçáo da resistência de média e longa duração $[35,36]$. A consubstanciar este papel de preditor da performance, estudos realizados com triatletas e corredores fundistas de elite revelaram elevadas correlaçóes entre o limiar anaeróbio e a prestação competitiva $[30,37,38]$.

O Limiar anaeróbio ventilatório $\left(\operatorname{Lan}_{\text {vent }}\right)$, mensurado emtermos percentuaisdo $\mathrm{VO}_{2 \max }$ ' registrou um valor médio de $85,4 \%$, reforçando os dados encontrados em estudos anteriores realizados com triatletas e corredores de elite de diferentes distâncias [39-42]. Em linha de convergência, refere-se que a uma intensidade de esforço entre $80-88 \% \mathrm{VO}_{2 \max }$, atletas que possuem limiar anaeróbio inferior deplecionam uma maior taxa de glicogénio muscular, o que se traduz numa concentração de lactato duas vezes superior a verificada em atletas com um registo de steady-state do lactato mais elevado [43].

Todavia, perante o fenómeno conflitual da determinação do limiar anaeróbio pelo método ventilatório, é provável que a comparação dos nossos resultados com outros estudos que avaliaram o respectivo indicador em triatletas, apresente limitaçóes devido ao fato de terem sido adotadas diferentes metodologias para sua determinação. Destarte, os pressupostos de determinação do ponto de inflexão do rácio $\mathrm{VE} / \mathrm{VO}_{2}$ e respectivo protocolo de determinaçáo do $\operatorname{Lan}_{\text {vent }}$, a performance durante o segmento da corrida é provavelmente o fator de maior importância no sucesso de uma prova de triathlon, sendo a sua correlação com o tempo total de prova significativamente elevada [7].

\section{Conclusão}

O presente estudo assevera a homogeneidade dos resultados relativos às variáveis antropométricas e fisiológicas entre triatletas portugueses e atletas internacionais de triathlon e das respectivas modalidades singulares referenciados na literatura, apesarde a capacidade de força explosiva,evidenciada nos saltos SE e SCM, mostrar-se reduzida. Os triatletas revelaram di- ferenças bilaterais e desequilíbrios na razão I/Q na avaliação dos parâmetros isocinéticos da força muscular nos membros inferiores, embora estejam os respectivos valores inseridos nos critérios considerados adequados.

\section{Referências}

1. Neumayr G, Pfister R, Mitterbauer G, H, Joanidis $\mathrm{M}$, Hoertnage H. Short-term effects of prolonged strenuous endurance exercise on the level of haematocrit in amateur cyclist. International J Sports Med 2002;23:158-61.

2. Basset FA, Boulay MR. Specificity of treadmill and cycle ergometer tests in triathletes, runners and cyclists. EurJ ApplPhysiol 2003;81:214-21.

3. Bentley DJ, McNughton LR, Lamyman R, Roberts SP. The effects of prior incremental cycle exercise on the physiological responses during incremental running to exhaustion: relevant for sprint triathlon performance. J Sports Sci 2003;21:29-38.

4. Hue O. Prediction of drafted-triathlon race time from submaximal laboratory testing in elite triathletes. Can J Appl Physiol 2003;28(4):547-60.

5. Palazzetti S, Margaritis I, Guezennec, CY. Swimming and cycling overload training in triathlon has no effect on running kinematics and economy. Int J Sports Med 2005;26:193-9.

6. Ackland TR, Blanksby B, Landers, G, Smith D. Anthropometric profiles of elite triathlete. J Sci Med Sport 1998;1:53-6.

7. Landers G, Blanksby B, Ackland TR, Smith D. Morphology and performance of world championship triathletes. Ann Hum Biol 2000;27(4):387-400.

8. Swain D. The influence of body mass in endurance bicycling. Med Sci SportExerc 1994;26(1):58-63.

9. Bogdanis G, Nevill M, Bobis L, Hacomy H. Contribution of phosphocreatine and aerobic metabolism to energy supply during repeated sprint exercise. J ApplPhysiol 1996;80(3):87684.

10. Driss T, Vandewalle H, Quivre J, Miller C, Monod $\mathrm{H}$. Effects of external loading on power in a squat jump on a force platform: a comparison between strength and power athletes and sedentary individuals. J Sports Sci 2001;19(2): 99-105.

11. Kellis E. Quantification of quadriceps and hamstrings antagonist activity. Sports Med 1998;25(1):37-62

12. Aagaard PS, Simonsen E, Magnusson S, Larsson B, Dyhre-Poulsen P. A new concept for isokinetic 
hamstring/quadriceps muscle strength ratio. American J Sports Med 1998;26(2):231-7.

13. Bosco C, Luhtanen P, Kpmi P. A simple method measurement of mechanical power in jumping. Eur J Applied Physiol 1983;50:273-82.

14. Billat V, Renoux J, Pinoteau J, Petit B, Koralsztein. Times to exhaustionat $100 \%$ of velocity at VO2max and modeling of the time-limit/velocity relationship in elite long distance runners. Eur J Appl Physiol 1994c;69:271-3.

15. Millet P, Dréano P, Bentley. Physiological characteristics of elite short and long distance triathletes. J Appl Physiol 2003;88:427-30.

16. Lucia A, Joyos H, Chicharro J. Physiological responses to professional road cycling: climbersvs time trialists. Int J Sports Med 2000;21:505-12.

17. Uçok K, Gokbel H, Okudan N. The load for wingate test: according to the body weight or lean body mass. Eur J Gen Med 2005;2(1):10-13.

18. Sleivert G, Rowlands, S. Physical and physiological factors associated with success in the triathlon. Sports Med 1996;22(1):58-63.

19. Titel K, Wutscherk H. Anatomical and anthropometric fundamentals of endurance. In: Shephard RJ, Astrand PO. Endurance in Sports. London: Blackwell; 1992.p. 35-45.

20. Komi P, Bosco C. Utilization of stored elastic energy in leg extensor muscles by man and women. Med Sci Sports 1978;10(4):261-5.

21. Ono M, Miyashita M, Asami T. Inhibitory effect of long distance running training on the vertical jump and other performances among aged males. In:Komi PV editor. Biomechanics V. Baltimore: MD University Park; 1976.

22. Luhtanen P, Komi PV. Segmental contribution to forces in vertical jump. Eur J ApplPhysiol 1978;38:181-8.

23. Peveler W, Bishop P, Smith J, Richardson M. Effects of training in aero position on anaerobic power output. J Exerc Physiol 2004;7(5):52-6.

24. Too D. The effect of trunk angle on power production in cycling. Res Q Exerc Sport 1997;65(4):308-15.

25. Heil DP, Derrick TK, Whitlesey S. The relationship between preferred and optimal positioning during submaximal cycle ergometry. Eur J Appl Physiol Occup Physiol 1997;75(2):160-5.

26. Koninckx E, Leemputte M, Hespel P. Effect of isocinetic cycling vs weight training on maximal power output and endurance performance in cycling. Eur J Appl Physiol 2010;109(4):699-708.

27. Garrandes F, Colson S, Pensini M, Seynnes O, Legros P. Neuromuscular fatigue profile in endu- rance-trained and power-trained athletes. Med Sci Sports Exerc 2007;39(1):149-58.

28. Aagaard P, Simonsen EB, Beyer N, Larsson B, Magnusson SP. A new concept of isokinetic hamstring/quadriceps muscle strength ratio. Am J Sports Med 1998;26(2):231-7.

29. Brown L. Isokinetics in human performance. Champaign: Human Kinetics; 2000.

30. Denadai BS, Piçarro I, Russo. Consumo máximo de oxigénio e limiar anaeróbio determinados em testes de esforço máximo, na esteira rolante, bicicleta ergométrica e ergómetro de braço, em triatletas brasileiros. Rev Paul Educ Fís 1994;8(1):49-57.

31. Laursen PB, Rhodes C. Factors affecting performance in an ultra-endurance triathlon. Sports Med 2001;31(3):195-209.

32. Vercruyssen F, Brisswalter J, Hausswirth C, Bernard $\mathrm{O}$, Vallier JC. Influence of cycling cadence on subsequent running performance in triathletes. Med Sci Sports Exerc 2002;34(3):530-6.

33. Sleivert G, Rowlands D. Physical and physiological factors associated with success in the triathlon. Sports Med 1996;22(1):8-18.

34. Hue O. Prediction of drafted-triathlon race time from submaximal laboratory testing in elite triathletes. Can J Appl Physiol 2003;28(4):547-60.

35. Heck H, Mader A, Hess G, Muller R, Hollmann W. Justification of the $4 \mathrm{mmol} / \mathrm{l}$ lactate Threshold. Int J Sports Med 1985;6:117-30.

36. Pate RR, Macera CA, Bailey SP. Physiological, anthropometric and training correlates of running economy. Med Sci Sports Exerc 1992;24:1128-33.

37. Jones A, Doust J. The validity of the lactate minimum test for determination of the maximal lactate steady state. Med Sci Sports Exerc 1998;1299-306.

38. Hausswirth C, Lehénaff D. Physiological demands of running during long distance runs and triathlons. Sports Med 2001;31(9):679-89.

39. De Vito G, Sproviero E, Figura F. Decrease of endurance performance during Olympic triathlon. Int J Sports Med 1995;16:24-28.

40. Kumagai S, Nishizumi M, Tanaka K. Lactate threshold and distance running performance. J Human Ergology 1987;58(4):1281-4.

41. Billat V, Mille-Hamard L, Koralsztein J. The role of cadence on the VO2max slow component in cycling and running triathletes. Int J Sports Med1999; 20:429-37.

42. Nicholson R, Sleivert G. Indices of lactate threshold and their relationship with $10 \mathrm{~km}$ running velocity. Med Sci Sports Exerc 2001;33(2):339-42.

43. Brooks GA. Anaerobic threshold: review of the concept and directions for future research. Med Sci Sports Exerc 1985;17:6-21. 